\title{
Graphene Nanocoating: High Quality and Stability upon Several Stressors
}

Journal of Dental Research 2021, Vol. 100(10) I169-1177

(c) International \& American Associations for Dental Research 2021

Article reuse guidelines:

sagepub.com/journals-permissions DOI: 10.1 | 77//002203452| I024526

journals.sagepub.com/home/jdr

\author{
V. Rosa',2 (D), R. Malhotra' ${ }^{(1)}$, S.V. Agarwalla', J.L.P. Morin'2, E.K. Luong-Van', \\ Y.M. $\operatorname{Han}^{3}$, R.J.J. Chew', C.J. Seneviratne ${ }^{4}$, N. Silikas ${ }^{5}$, K.S. Tan ${ }^{1,2}$, C.A. Nijhuis ${ }^{6}$, \\ and A.H. Castro Neto ${ }^{2}$
}

\begin{abstract}
Titanium implants present 2 major drawbacks_-namely, the long time needed for osseointegration and the lack of inherent antimicrobial properties. Surface modifications and coatings to improve biomaterials can lose their integrity and biological potential when exposed to stressful microenvironments. Graphene nanocoating (GN) can be deposited onto actual-size dental and orthopedic implants. It has antiadhesive properties and can enhance bone formation in vivo. However, its ability to maintain structural integrity and quality when challenged by biologically relevant stresses remains largely unknown. GN was produced by chemical vapor deposition and transferred to titanium via a polymer-assisted transfer technique. GN has high inertness and did not increase expression of inflammatory markers by macrophages, even in the presence of lipopolysaccharides. It kept high coverage at the top tercile of tapered dental implant collars after installation and removal from bone substitute and pig maxilla. It also resisted microbiologically influenced corrosion, and it maintained very high coverage area and quality after prolonged exposure to biofilms and their removal by different techniques. Our findings show that GN is unresponsive to harsh and inflammatory environments and that it maintains a promising level of structural integrity on the top tercile of dental implant collars, which is the area highly affected by biofilms during the onset of implant diseases. Our findings open the avenues for the clinical studies required for the use of GN in the development of implants that have higher osteogenic potential and are less prone to implant diseases.
\end{abstract}

Keywords: corrosion, titanium, implant dentistry/implantology, nanotechnology, prostheses and implants, biocompatible materials

\section{Introduction}

Titanium implants have 2 major shortcomings: long time to integrate and lack of inherent antimicrobial properties. The first can be a negative factor for patient engagement in rehabilitation (Johannsen et al. 2012), whereas the latter allows the development of biofilm-associated implant diseases (Derks et al. 2016).

Surface coatings to improve implant integration can present unfavorable mechanical properties, posing a risk of delamination (Fares et al. 2020). Also, antibiotic surfaces may not present optimized release kinetics or be able to damage specific microbial strains, hence increasing the chances of resistant isolates (Derks et al. 2016). Graphene nanocoating (GN) is a promising platform for improving implantable materials (Xie et al. 2017). It is a single layer of carbon atoms arranged in a honeycomb structure and can be deposited onto actual-size dental and orthopedic implants (Morin et al. 2017; Xie et al. 2017). Titanium coated with GN is cytocompatible, has antiadhesive properties against early microbial attachment, and enhances bone formation in vivo (details in Appendix; Dubey et al. 2018; Agarwalla et al. 2019; Dubey et al. 2020).

Coatings are subjected to several stresses that challenge their integrity and decrease biological properties and that contribute to degradation, debonding, and failure (Roosjen et al. 2005; Pidhatika et al. 2012; Schönweger et al. 2020). Likewise, the environment from biofilms can negatively affect the stability and integrity of materials and induce microbiologically influenced corrosion (MIC; see Appendix and Souza et al. 2010a, 2010b). Although GN has shown promising potential to improve implants, its clinical translation depends on 1) its ability to withstand stresses from installation and cleaning, 2) its inability to elicit relevant inflammatory/foreign body reactions that can compromise tissue homeostasis, and 3) its ability to maintain its structural integrity characteristics when exposed to harsh microenvironments. Here, we show that GN presents high structural stability and does not induce inflammatory

'Faculty of Dentistry, National University of Singapore, Singapore ${ }^{2}$ Centre for Advanced 2D Materials and Graphene Research Centre, National University of Singapore, Singapore

${ }^{3}$ Department of Chemistry, National University of Singapore, Singapore ${ }^{4}$ National Dental Centre Singapore, SingHealth, Singapore

${ }^{5}$ Division of Dentistry, School of Medical Sciences, University of Manchester, Manchester, United Kingdom

${ }^{6}$ Department of Molecules and Materials, Faculty of Science and Technology, University of Twente, Enschede, Netherlands

A supplemental appendix to this article is available online.

Corresponding Author:

V. Rosa, Faculty of Dentistry, National University of Singapore, 9 Lower Kent Ridge Road, Singapore I 19082, Singapore.

Email: denvr@nus.edu.sg 
reactions when challenged by different biologically relevant stresses.

\section{Materials and Methods}

Detailed methodologies and statistical analysis are described in the Appendix.

\section{Sample Preparation}

Graphene was prepared by chemical vapor deposition (CVD) in a class 1,000 cleanroom by the pyrolysis of $\mathrm{CH}_{4}(16 \mathrm{sccm}$, $1 \mathrm{~h})$ in a quartz tube containing 35- $\mu \mathrm{m}$-thick copper film (Graphene Platform; Morin et al. 2017). Afterward, the graphene film was transferred to Ti-6Al-4V disks (12-mm diameter, 1-mm thickness; United Performance Metals) or a dental implant (tapered, TiU RP $4.0 \times 10 \mathrm{~mm}$ [Nobel Biocare]; Morin et al. 2017; Dubey et al. 2020). The GN structural characteristics were characterized by Raman spectroscopy (CRM 200; Witec) for the identification of $\mathrm{G}$ peak $\left(\sim 1,587 \mathrm{~cm}^{-1}\right.$, stretching of the $\mathrm{C}-\mathrm{C}$ bond in graphitic materials $)$, D peak $\left(\sim 1,340 \mathrm{~cm}^{-1}\right.$, risen if defects are present), and 2D peak (2,500 to $2,800 \mathrm{~cm}^{-1}$, present in $s p^{2}$ carbon materials and used to determine the number of layers of graphene). We also mapped the full width at half maximum of $2 \mathrm{D}$ peak $\left(\mathrm{FWHM}_{2 D}\right)$ obtained from the curve defined by counts as a function of peak intensity (OriginPro 2020b; OriginLab) and the ratio obtained from the intensities of $2 \mathrm{D}$ and $\mathrm{G}$ peaks $\left(\mathrm{I}_{2 D} / \mathrm{I}_{G}\right)$ for the characterization of GN layers. The mappings built from the ratio of $2 \mathrm{D}$ and $\mathrm{G}$ peaks $\left(\mathrm{I}_{D} /\right.$ $\mathrm{I}_{G}$ ) were used to characterize the defect population. Samples were imaged with atomic force microscope (Bruker AXS). Uncoated samples were the control set.

\section{Integrity of GN upon Friction Stresses from Implant Installation}

The structural integrity of the GN on implant installation (tapered, TiU RP $4.0 \times 10 \mathrm{~mm}$; Nobel Biocare) was evaluated before and after the installation of implants in bone substitutenamely, solid rigid polyurethane (SRP fulfilling ASTM F-1839-08; Sawbones) - and the maxilla from a pig carcass, according to an actual clinical surgical protocol. The implants were installed at $35 \mathrm{~N} \cdot \mathrm{cm}$ (Implantmed; W\&H), and the working length of the burs was increased by $2 \mathrm{~mm}$ to ensure that the implant collar (the part that is first exposed to biofilm on the onset of implant diseases) was inserted fully into the substrate. After $30 \mathrm{~s}$ from insertion, the implants were removed by reverse rotation (bone) or by fracturing the SRP and analyzed with the Raman spectroscope.

\section{Cytocompatibility of GN and Its Potential to Induce or Amplify Inflammatory Response}

The cytocompatible character of GN was studied by monitoring the attachment, morphology, proliferation, and viability of RAW 246.7 macrophages. Following this, macrophages were cultured on GN coated and control samples and were treated with lipopolysaccharide (LPS) from Escherichia coli (SigmaAldrich), and the expression of proinflammatory markers and cytokines was quantified by polymerase chain reaction and enzyme-linked immunosorbent assay. The stability of the GN was characterized with Raman spectroscopy before and after being exposed to macrophages stimulated with LPS for $7 \mathrm{~d}$.

\section{GN Resistance to MIC}

Streptococcus mutans (S. mutans) was used to examine MIC, as it has been isolated in high numbers around failing implants. Here, we studied the effects of prolonged exposure to $S$. mutans on the electrochemical properties using linear polarization resistance curves and electrochemical impedance spectroscopy. The GN potential to resist MIC was studied by supplementing the culture with $0.1 \%$ and $1 \%$ sucrose. Confocal laser scanning microscopy with LIVE/DEAD staining, glycolytic $\mathrm{pH}$ drop assay, and quantitative real-time polymerase chain reaction were performed to ensure similar corrosive microenvironments. Finally, the GN's structural and lattice stability was determined per Raman spectroscopy following biofilm exposure.

\section{GN Stability after Prolonged Exposure to Biofilms and Their Removal}

Next, we characterized the structural integrity of GN on titanium alloy after prolonged exposure to microorganisms and removal procedures. Here, we used Candida albicans as this species 1) has been identified in implant sites (Schwarz et al. 2015); 2) can produce different types of acids (pyruvate, acetate, $\alpha$-ketoglutarate, and malate); and 3) presents $\mathrm{H}^{+}$-ATPase in the plasma membrane, which actively pumps out protons to generate an electrochemical gradient that can contribute to microenvironment net acidification (Manavathu et al. 1999; Klinke et al. 2009). The GN's structural characteristics were characterized by Raman spectroscopy and AFM up to $7 \mathrm{~d}$ after biofilm exposure and removal by saponification reaction (SR) or liquid shear forces and ultrasonication (chaotic turbulent flow $[\mathrm{TF}])$.

\section{Statistical Analysis}

All tests were performed in triplicates (unless stated otherwise) with a preset significance of $5 \%$. The statistical tests used in analyses were 1-sample $t$ test (for comparisons between control and graphene-nanocoated samples) and paired $t$ test (for comparisons of same-group samples before and after exposure to biofilms).

\section{Results}

\section{GN Integrity after Simulated Implant Installation}

To evaluate the ability of GN to withstand frictional forces from implant installation, we coated the collar of an implant 


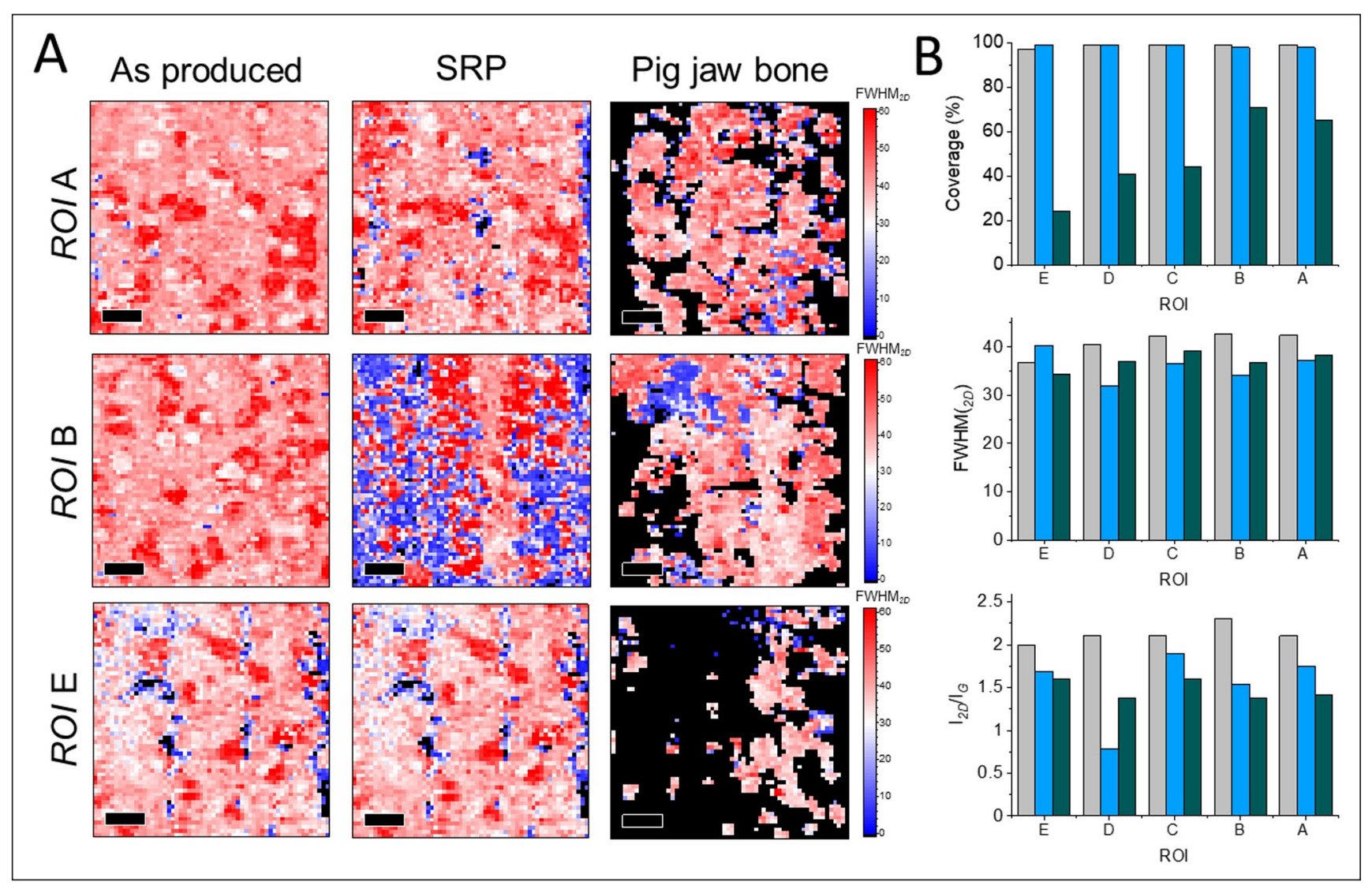

Figure I. The collar of tapered implants were coated with graphene nanocoating $(\mathrm{GN})$ and installed in solid rigid polyurethane (SRP) or pig jaw. (A) The mappings for $2 \mathrm{D}$ full width at half maximum $\left(\mathrm{FWHM}_{2 \mathrm{D}}\right.$ ) obtained from 5 regions of interest at different points running vertically down the collar (ROls; see Appendix Fig. 2B). GN coverage was not affected by SRP. (B) The coverage decreased by $35 \%$ after installation and removal by reverse rotation from jaw bone (ROls A and B). (A) Scale bar $=5 \mu \mathrm{m}$, area of Raman mappings is $900 \mu \mathrm{m}^{2}$. (B) Gray, as produced; blue, after removal from SRP; green, after removal from jaw bone.

and installed it fully in SRP or pig maxilla. The implants were removed from the SRP by brittle fracture (Appendix Fig. 2A) and from the bone by reverse rotation. Raman mappings were rendered from 5 regions of interest (ROIs; Appendix Fig. 2B). There were no significant differences in GN coverage and structural characteristics arising from the insertion in SRP. The mappings obtained after implant removal from bone showed that ROIs A and B kept at least $65 \%$ of coverage whereas ROI C, D, and E kept 50\%, 40\%, and $20 \%$, respectively. The $\mathrm{I}_{2 D} / \mathrm{I}_{G}$ confirmed the presence of layers of graphene on the implant surface after the installation and removal from bone (Fig. 1A, B; Appendix Figs. 3, 4).

\section{GN Is Cytocompatible and Does Not Exacerbate Inflammatory Response}

We assessed GN cytocompatibility and early attachment dynamics using immune macrophages (RAW 246.7). Cell attachment efficiency and viability on GN were similar to the control samples (Fig. 2A). To ensure that the cell attachment on the GN was not solely dictated by the titanium alloy, we also evaluated with $\mathrm{SiO}_{2}$, which is less favorable for cell anchorage than CVD-grown graphene (Kalbacova et al. 2010).
At 10 min, GN was able to improve cell attachment on $\mathrm{SiO}_{2}$ (Fig. 2A). The LIVE/DEAD assay and scanning electron microscopy images confirmed that GN did not induce relevant cell death or abnormal morphology (24 h; Fig. 2B-D). Following this, we investigated whether GN could promote or enhance inflammatory response on macrophages while maintaining its structural integrity. Macrophages cultured on GN presented similar genetic expression of inflammatory markers when compared with the control, regardless of the treatment with LPS (Fig. 2E, F). The Raman analysis showed that the GN remained mostly unaffected by the presence of macrophages treated with LPS and that the defective areas were limited to a few micrometers in size (green arrow, Fig. 2G). The stresses generated from cell removal and preparation for Raman characterization could not be discarded as a cause for defect creation after cell culture.

\section{GN Is Not Affected by MIC}

We investigated whether biofilms could negatively affect GN's electrochemical stability, thereby inducing its degradation. First, we obtained potentiodynamic polarization curves from samples exposed to $S$. mutans for up to $8 \mathrm{~d}$ (Fig. 3A). The 


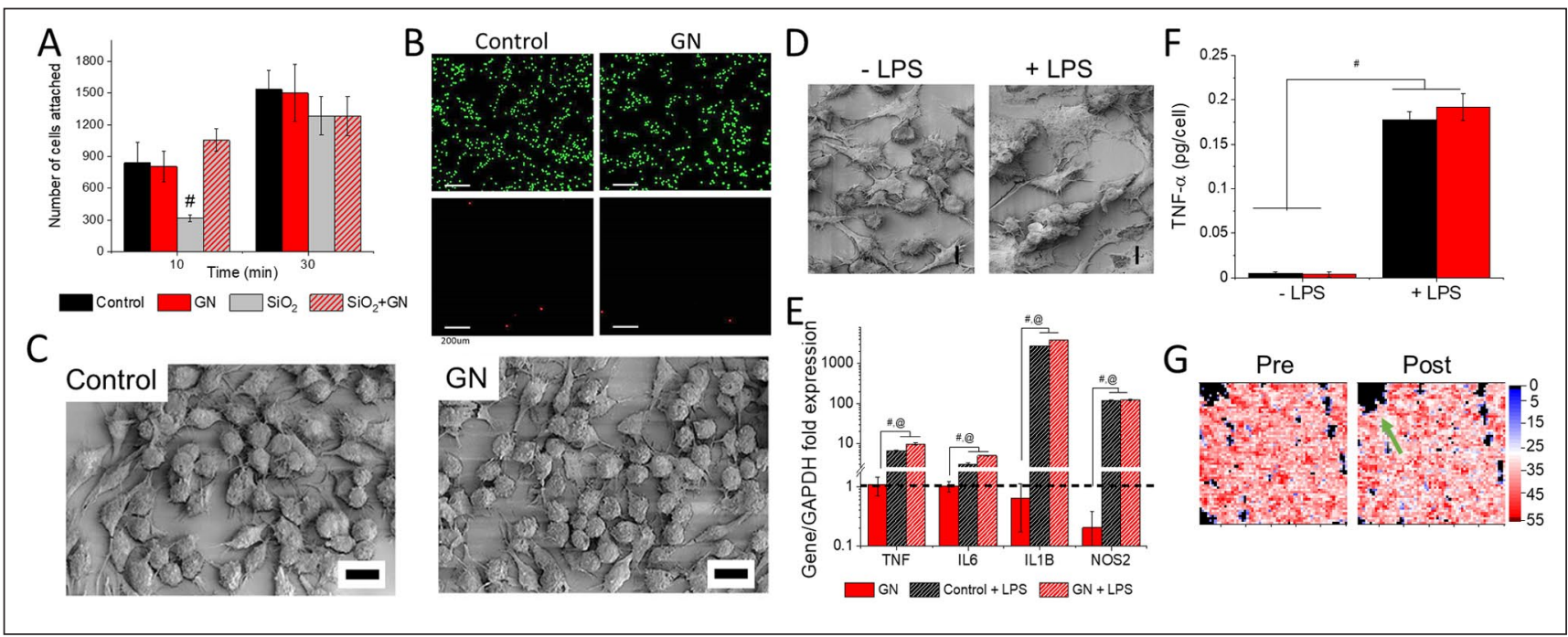

Figure 2. GN is cytocompatible and does not induce or exacerbate inflammatory responses in vitro. (A) RAW 246.7 attachment rate on graphene nanocoating (GN) on titanium was similar to that observed on uncoated titanium (control). (B, C) The LIVE/DEAD assay and scanning electron microscopy show that GN did not induce cell death or abnormal cell morphology. (D-F) The morphology, expressions of inflammatory markers, and TNF $\alpha$ expressions of cells on GN were similar to those observed on the control regardless of the treatment with lipopolysaccharide (LPS), except for GN/NOS2. (G) The Raman mappings of 2D full width at half maximum show that GN remained mostly unaffected by the presence of macrophages treated with LPS for $7 \mathrm{~d}$. Few defective areas were observed after the removal of GN, but these were limited to few micrometers in size (green arrow). Values are presented as mean \pm SD. $\# P<0.05$; @ $<0.05$ compared with control no LPS (dashed line). Scale bars $=200 \mu \mathrm{m}$ in panel B, $10 \mu \mathrm{m}$ in panels $C$ and D. Area of Raman mappings is $900 \mu \mathrm{m}^{2}$. Dashed line in E represents Control samples.

polarization sweeps of the control samples showed a significant cathodic shift of corrosion potential when compared with the negative control (no biofilm; $E_{\text {corr }}$ shifted from $-0.334 \mathrm{~V}$ to $-0.402 \mathrm{~V}$ at $1 \mathrm{~d}$ and $-0.252 \mathrm{~V}$ to $-0.412 \mathrm{~V}$ at $8 \mathrm{~d}$ ), whereas no significant changes in $E_{\text {corr }}$ were observed for GN $\left(E_{\text {corr }}\right.$ at $1 \mathrm{~d}$ $-0.242 \mathrm{~V}$ to $-0.247 \mathrm{~V}$ and $-0.337 \mathrm{~V}$ to $-0.348 \mathrm{~V}$ at $8 \mathrm{~d}$, preand postexposure to biofilm, respectively). Next, sucrose was added to the brain-heart infusion broth, as increases in sucrose are often associated with higher bacteria metabolic activity that facilitates acid production and can promote corrosion of titanium (Koike and Fujii 2001; Prando et al. 2017). The Table shows that controls exposed to $S$. mutans cultured with $0.1 \%$ sucrose presented significantly increased $j_{\text {corr }}(0.676 \pm 0.030$ $\left.\mu \mathrm{A} / \mathrm{cm}^{2}\right)$ and decreased polarization resistance $(R p=0.033 \pm$ $0.014 \mathrm{M} \Omega$ ). Exposure to $1 \%$ sucrose promoted an obvious cathodic shift in $E_{\text {corr }}$ (from -0.342 to $-0.594 \mathrm{~V}$ ), accompanied by an increase in $j_{\text {corr }}\left(0.163 \pm 0.041 \mu \mathrm{A} / \mathrm{cm}^{2}\right)$. Notably, the GN's electrochemical properties were not affected by $S$. mutans and supplementation with sucrose, and there were no significant changes in the polarization curves of the GN that presented an anodic open circuit potential, a relatively constant corrosion potential and corrosion current density $\left(E_{\text {corr }} \sim 0.2 \mathrm{~V} ; j_{\text {corr }}\right.$ $\sim 0.09 \mu \mathrm{A} / \mathrm{cm}^{2}$ ).

Electrochemical impedance spectroscopy was done to assess corrosion resistance via the application of a small sinusoidal AC perturbation $( \pm 10 \mathrm{mV})$ at open circuit potential, which enabled us to study small changes in the electrochemical characteristics. First, the negative imaginary and real parts of impedance were plotted to yield a Nyquist plot. The straight slope of the Nyquist plot implies a double-layer capacitance at the sample surface and electrolyte interface. The GN samples presented a more vertical slope, suggesting lower capacitance and superior corrosion resistance as compared with the control (uncoated). Furthermore, the impedance data were fit in a Randle's electrical equivalent circuit (constant phase element in parallel with charge transfer resistance, which is in series with solution resistance), and GN presented a higher charge transfer resistance $\left(R_{\mathrm{ct}}\right)$, indicating superior resistance to electron transfer and electrochemical reactions at the surface as compared with control counterparts. Finally, Raman showed that GN integrity was not affected (Appendix Fig. 7, Appendix Table 3), suggesting that this coating has thermodynamic and mechanical stability, electrochemical resistance, and corrosionprotective behavior against bacterial metabolic products.

\section{GN Has High Structural Stability after Prolonged Exposure to Biofilms and Their Removal}

The stability and structural integrity of GN were studied by Raman obtained before and after $7 \mathrm{~d}$ of exposure to microorganisms and their removal via SR or chaotic TF. Herein, we studied the effects of transfer procedures $\left(2, \mathrm{GN}_{2 T} ; 5, \mathrm{GN}_{5 T}\right)$ to determine possible loss of GN due to handling and the removal of cells.

The coverage loss on samples cleaned via SR was negligible $(<2 \%)$ for $\mathrm{GN}_{2 T}$ and $\mathrm{GN}_{5 T}$ (Fig. 4A, B), with the lowest coverage being $97.5 \%$ for $\mathrm{GN}_{2 T}$ at $5 \mathrm{~d}$ (Appendix Table 4). The presence of multilayer graphene for both groups throughout the study was confirmed by the $\mathrm{FWHM}_{2 D}>30$ and $\mathrm{I}_{2 D} / \mathrm{I}_{G}<1.2$ (Fig. 4C). The $\mathrm{I}_{D} / \mathrm{I}_{G}$ indicates that microbes and SR introduced a very small defect population in $\mathrm{GN}_{2 T}$ and $\mathrm{GN}_{5 T}$ (Fig. $4 \mathrm{C}$, Appendix Fig. 11). The Raman mappings obtained after TF 


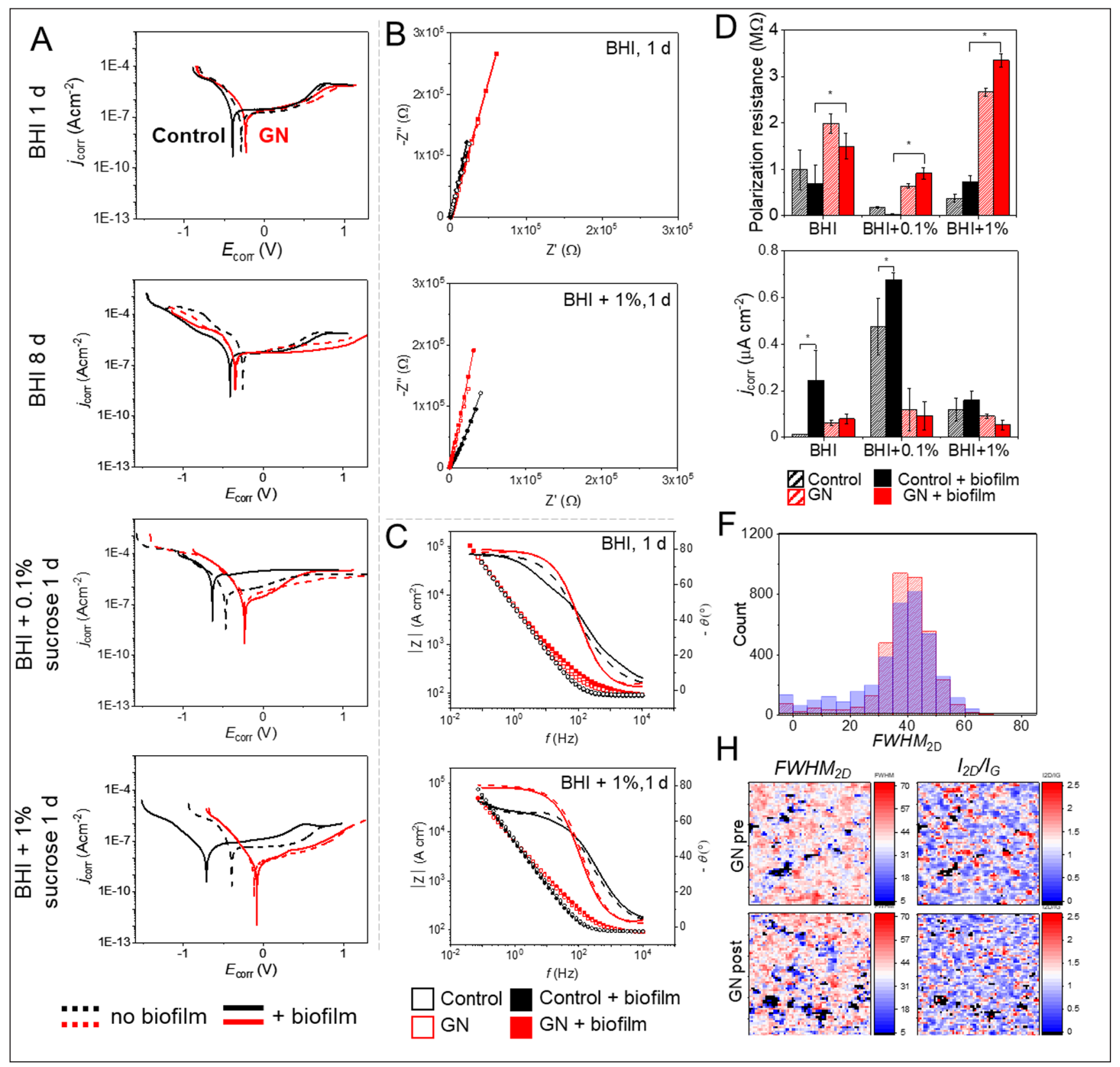

Figure 3. Potentiodynamic polarization sweeps of uncoated control (black) and graphene nanocoating (GN; red) samples in the presence (solid lines) and absence (dashed lines) of Streptococcus mutans biofilm in various culture conditions. (A) The GN samples show no change in the $E_{\text {corr, }}$ suggesting its electrochemical stability over control samples, which showed significant cathodic shift in the presence of biofilm. Corrosion dynamics of samples were assessed through electrochemical impedance spectroscopy after culturing biofilms in brain-heart infusion (BHI) media alone and supplemented with $1 \%$ sucrose for $24 \mathrm{~h}$. (B) Nyquist plots show a relatively more vertical slope of GN samples (solid and open red squares indicate the presence and absence of biofilm, respectively), implying its lower capacitance over control samples (solid and open black squares). Bode impedance and phase angle plots show no changes in impedance data with either sample. (C) GN samples displayed a phase angle close to $-90^{\circ}$ over a range of intermediate frequencies as compared with controls. Tafel analysis of potentiodynamic polarization sweeps illustrate significantly (D) higher polarization resistance and (E) lower corrosion current density of GN samples (red) as compared with uncoated controls (black) in the presence of biofilm, indicating its higher resistance toward microbiologically influenced corrosion. The stability of GN was checked with Raman spectroscopy. (F) The histograms show no loss of total pixels before (red) and after (blue) exposure of biofilms at a particular 2D full width at half maximum (FWHM ${ }_{2 D}$ ). (G) The FWHM $2 D$ and $\mathrm{I}_{2 D} / \mathrm{I}_{G}$ heat maps obtained from Raman mappings of $\mathrm{GN}$ samples demonstrate minimal graphene loss postexposure (black pixels indicate absence of graphene). One-way analysis of variance was performed to analyze difference between control and GN samples in the presence or absence of biofilm. Values are presented as mean $\pm S D$ among 3 independent samples. $* P<0.05$. Area of the mappings $=900 \mu \mathrm{m}^{2}$.

showed a loss of approximately $10 \%$ of $\mathrm{GN}_{2 T}$ and $<2 \%$ for $\mathrm{GN}_{5 T}$, suggesting higher structural integrity for the latter (Fig. 4A, C; Appendix Figs. 9 and 11, Appendix Table 5). The micrographs show an area where the $\mathrm{GN}_{2 T}$ detached from the substrate, whereas the $\mathrm{GN}_{5 T}$ kept the characteristic ripples and wrinkles throughout the study (Fig. 4B, Appendix Fig. 9). 
Table. Tafel Analysis of Linear Polarization Resistance Data from Control and GN before and after 24-h Exposure to Streptococcus mutans Cultured in Different Conditions.

\begin{tabular}{|c|c|c|c|}
\hline S. mutans Culture Condition: Biofilm Exposure & $E_{\text {corr }}, \mathrm{V}$ & Corrosion Rate, mm/y & OCP, V \\
\hline \multicolumn{4}{|l|}{ BHI } \\
\hline \multicolumn{4}{|l|}{ Control } \\
\hline Before & $-0.334 \pm 0.062$ & $0.0003 \pm 0.0004$ & $-0.099 \pm 0.079$ \\
\hline After & $-0.402 \pm 0.046^{\mathrm{a}}$ & $0.0012 \pm 0.0019^{\mathrm{a}}$ & $-0.048 \pm 0.077$ \\
\hline \multicolumn{4}{|l|}{ GN } \\
\hline Before & $-0.242 \pm 0.094^{b}$ & $0.00054 \pm 0.0583$ & $0.249 \pm 0.100^{\mathrm{b}}$ \\
\hline After & $-0.247 \pm 0.080^{b}$ & $0.00047 \pm 0.0605$ & $0.209 \pm 0.108^{b}$ \\
\hline \multicolumn{4}{|l|}{ BHI $+0.1 \%$ sucrose } \\
\hline \multicolumn{4}{|l|}{ Control } \\
\hline Before & $-0.512 \pm 0.064$ & $0.0058 \pm 0.003$ & $-0.009 \pm 0.098$ \\
\hline After & $-0.627 \pm 0.008^{\mathrm{a}}$ & $0.0236 \pm 0.002^{\mathrm{a}}$ & $-0.051 \pm 0.016^{\mathrm{a}}$ \\
\hline \multicolumn{4}{|l|}{ GN } \\
\hline Before & $-0.242 \pm 0.04 \mathrm{I}^{\mathrm{b}}$ & $0.0002 \pm 0.000^{\mathrm{b}}$ & $0.114 \pm 0.04 I^{b}$ \\
\hline After & $-0.223 \pm 0.014^{b}$ & $0.0007 \pm 0.000^{b}$ & $0.207 \pm 0.112^{b}$ \\
\hline \multicolumn{4}{|l|}{ BHI + I\% sucrose } \\
\hline \multicolumn{4}{|l|}{ Control } \\
\hline Before & $-0.342 \pm 0.089$ & $0.0011 \pm 0.003$ & $0.092 \pm 0.043$ \\
\hline After & $-0.594 \pm 0.187^{a}$ & $0.0013 \pm 0.00 \mathrm{I}^{\mathrm{a}}$ & $-0.006 \pm 0.017^{a}$ \\
\hline \multicolumn{4}{|l|}{ GN } \\
\hline Before & $-0.226 \pm 0.048^{b}$ & $0.0007 \pm 0.000^{b}$ & $0.179 \pm 0.019 b$ \\
\hline After & $-0.182 \pm 0.132^{\mathrm{a}, \mathrm{b}}$ & $0.0004 \pm 0.000^{b}$ & $0.209 \pm 0.1 I^{a, b}$ \\
\hline
\end{tabular}

Values are presented as mean \pm SD of 3 independent samples.

$\mathrm{BHI}$, brain-heart infusion; $E_{\text {corr }}$ corrosion potential; $\mathrm{GN}$, graphene nanocoating; OCP, open circuit potential. ${ }^{a} P<0.05$. Paired $t$ test performed to analyze the difference in each group for presence or absence of biofilm.

${ }^{\mathrm{b} P}<0.05$. One-sample $t$ test performed to analyze the difference between the control and graphene groups.

Notably, biofilms were unable to introduce major defects into the GN lattice, as confirmed by the $\mathrm{I}_{D} / \mathrm{I}_{G}<0.3$ for all groups (Fig. 4B, Appendix Figs. 9 and 11, Appendix Tables 4 and 5). The scatterplots $\left(\mathrm{I}_{2 D} / \mathrm{I}_{G}\right.$ vs. $\left.\mathrm{FWHM}_{2 D}\right)$ show a similar distribution, confirming that neither the microorganisms nor the cleaning procedures negatively affect GN quality (Fig. 4D, Appendix Fig. 12).

\section{Discussion}

Titanium implants present 2 major shortcomings: the long time taken for effective integration and no inherent antimicrobial properties (Huang et al. 2001; Chouirfa et al. 2019; Fares et al. 2020). GN on titanium alloy has promising antiadhesive properties and can increase bone formation (Agarwalla et al. 2019; Dubey et al. 2020). Nonetheless, clinical translation of GN depends on its ability to endure a variety of stresses. Here, we show that GN does not induce or exacerbate inflammatory processes and that it maintains its quality, electrochemical, and structural characteristics when confronted by physicochemical stress and microbial-rich environments.

Friction can promote partial delamination of carbon-based coatings from titanium when scratched at loads $>400 \mathrm{mN}$ (Huang et al. 2001). We were unable to perform peeling tests to measure direct adhesion strength since GN thickness is confined to a few atomic layers. Instead, we characterized GN on bone-level tapered implants before and after the installation and removal from SRP and maxilla. No structural changes were observed in the GN when inserted into the SRP (Fig. 1A,
B), but we observed a decrease in coverage area downward from ROI $\mathrm{C}$ after installation and removal from the bone. However, we cannot discard, as a reason for GN loss, potential additional stresses created by the reverse rotations used to retrieve the implant. The decrease in coverage area may compromise GN availability as anchor points to osteoblasts, possibly decreasing its potential to enhance osteogenesis (Luong-Van et al. 2020). Nevertheless, some strategies may be used to improve GN attachment to titanium alloys, such as thermal treatment steps or an increased number of transfer procedures (Gu et al. 2018; Agarwalla et al. 2019). Notably, ROIs $A$ and $B$ maintained high coverage and structural integrity (Fig. 1B). This can be advantageous to prevent the initial microorganism colonization on the collar of tapered implants (Dubey et al. 2018). It is worth highlighting that the collars of implants installed at the tissue level are not subjected to such extreme surgical friction stresses; hence, GN integrity may be challenged by other means, as discussed later.

Implant materials must not compromise tissue homeostasis. We have seen that cell morphology, attachment rate, and proliferation on GN were similar to the control (Fig. 2A-D). The implantation of biomaterials can cause acute inflammatory response that is typically self-contained and resolves rapidly. However, biomaterials may elicit undesired severe chronic inflammation that triggers the body to accelerate their dissolution or resorption or even promote their rejection (Anderson et al. 2008). With the establishment of a biomaterial-tissue interface, several chemoattractant signals produced by macrophages (e.g., tumor necrosis factor $\alpha$ ) are released to recruit 


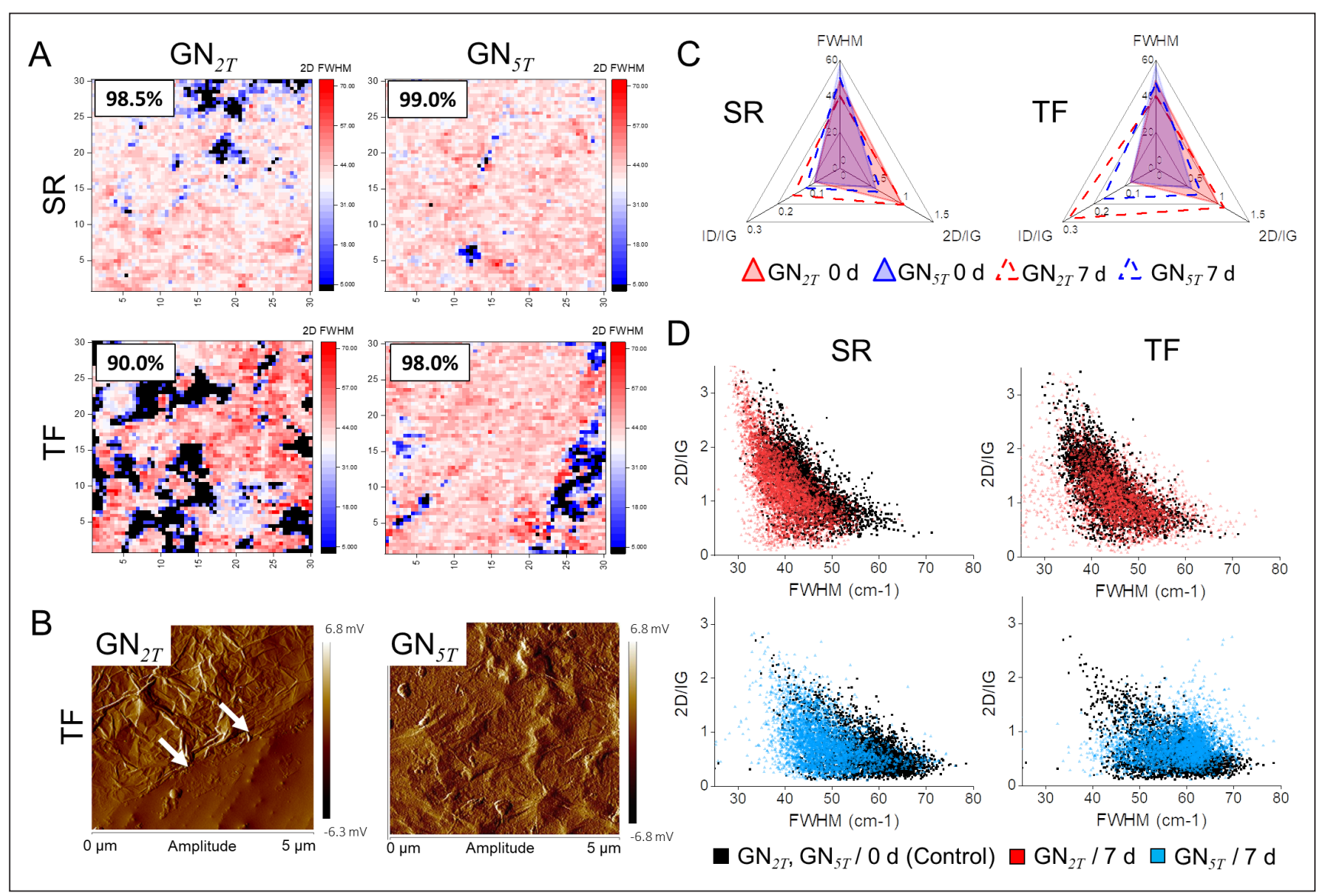

Figure 4. Raman characterization of $\mathrm{GN}_{2 T}$ and $\mathrm{GN}_{5 T}$ after being exposed to biofilms for $7 \mathrm{~d}$ and removal via saponification reaction (SR) or chaotic turbulent flow (TF). For SR, the Raman mappings confirmed high coverage yields ( $\geq 98 \%$ ) with high-quality multilayer graphene for both groups. (A) For $\mathrm{TF}$, the coverage yield was $90 \%$ for $\mathrm{GN}_{2 T}$ and $98 \%$ for $\mathrm{GN}_{5 T}$. (B) The white arrows indicate loss of graphene coverage on the atomic force micrographs obtained from $\mathrm{GN}_{2 T}$, whereas $\mathrm{GN}_{5 T}$ remained intact after microbial challenge and removal by TF (atomic force microscopy; area of the micrographs, $25 \mu \mathrm{m}^{2}$ ). (C) There were no significant changes in the structural characteristics of $\mathrm{GN}_{2 T}$ (red) and $\mathrm{GN}_{5 T}$ (blue) at $7 \mathrm{~d}$ (dashed triangles) as compared with $0 \mathrm{~d}$ (before exposure to biofilms). Although TF increased $I_{D} / I_{G}$, the values were $<0.3$, indicating high-quality graphene. (D) The scatter plots of $I_{2 D} /$ $\mathrm{I}_{G}$ and $2 \mathrm{D}$ full width at half maximum $\left(F W H M_{2 D}\right)$ obtained before $(0 \mathrm{~d})$ and after $(7 \mathrm{~d})$ biofilm growth and removal present similar patterns, indicating that the exposure to microbials and their removal had almost no impact on the structural and morphologic properties of the graphene coating. Black squares were obtained from the GN before its exposure to biofilms ( $0 \mathrm{~d}$ ); red and blue squares were obtained from $\mathrm{GN}$ after being exposed to biofilms for $7 \mathrm{~d}$, followed by removal procedures. 2T, 2 transfer procedures; $5 \mathrm{~T}, 5$ transfer procedures. Area of the Raman mappings: $900 \mu m^{2}$. GN, graphene nanocoating.

macrophages to the wound site (Broughton et al. 2006). Macrophage fusion to form foreign-body giant cells on biomaterial surfaces is material dependent (Jones et al. 2012). Our GN does not induce the overexpression of typical inflammatory markers by macrophages even with LPS stimulation (Fig. $2 \mathrm{E}, \mathrm{F})$, which is known to trigger the ample secretion of cytokines from macrophages such as TNF- $\alpha$ (Tan et al. 2007). Finally, GN remained stable after the culture and removal of macrophages stimulated with LPS for $7 \mathrm{~d}$ (Fig. 2G).

Microorganisms can reduce the environmental $\mathrm{pH}$ and oxygen concentration around implants (Tan et al. 2007), challenging the passivation of titanium alloys and thus promoting its deterioration (Fretwurst et al. 2016). Herein, we have studied GN electrochemical stability and structural integrity after being challenged with $S$. mutans, which can accelerate corrosion and decrease corrosion resistance of titanium alloys
(Mabilleau et al. 2006; Fukushima et al. 2014; Qu et al. 2014). Overall, GN presented superior electrochemical characteristics when compared with the control, as confirmed by higher polarization resistance and lower corrosion rates (Fig. 3, Table). The ability of GN on titanium alloy to resist MIC is similar to the anticorrosion potential of CVD-grown graphene films on arbitrary materials produced with alternative protocols and transferred via different methods (Duan 2019). Hence, it is credible that the GN ability to resist MIC is intrinsically related to the obtention of a homogenous graphene coating than to the production and transfer methods. The GN resistance to corrosion can be related to the small geometric pore size of the hexagonal $s p^{2}$ atomic arrangement, which does not allow the penetration of molecules (Berry 2013) and the high electron density ( $\pi$ cloud) that creates a repelling field (Bunch et al. 2008). The Raman characterization did not show relevant changes in GN 
coverage and structural characteristics after being exposed to S. mutans cultured with different concentrations of sucrose (Appendix Fig. 7), confirming its stability when challenged by an acidic bacterial microenvironment.

Next, we tested whether prolonged exposure to biofilm could compromise GN's structural integrity. Notably, GN maintained a coverage area of $98 \%$ after $7 \mathrm{~d}$ of exposure to microbes and removal with SR. It is feasible that the biofilms per se were not able to introduce a significant defect population that could induce coating degradation or detachment. To challenge GN stability, samples were subjected to intense liquid shear forces created by chaotic TF, followed by ultrasonication. Figure $4 \mathrm{~A}-\mathrm{C}$ and Appendix Tables 4 and 5 show that $\mathrm{GN}_{2 T}$ had lower $\mathrm{FWHM}_{2 D}$ than $\mathrm{GN}_{5 T}(39.8$ vs. 46.8$)$ and lost $10 \%$ of coverage at $7 \mathrm{~d}$. These discrepancies could be attributed to the relationship between defect population and the number of GN transfer procedures (Appendix Fig. 1). Molecular dynamics simulations have shown that increases in the number of graphene layers do not enhance mechanical properties and that graphene fracture stress is insensitive to the number of layers ranging from 1 to 7 (Zhang and Gu 2013). We hypothesized that the higher stability of $\mathrm{GN}_{5 T}$ could be related to the occlusion of lattice defects by the added layers bringing homogeneity to the nanocoating. This could prevent ingress of liquids through defects between the layers, decreasing the likelihood of moisturization of the substrate and preventing GN detachment (Appendix Fig. 13). Our rationale was supported by Raman, which confirmed higher coverage and fingerprint ratios consistent with the presence of more layers and a lower defect population in $\mathrm{GN}_{5 T}$ versus $\mathrm{GN}_{2 T}$ (Fig. 4C, Appendix Fig. 11). Despite the higher loss observed for $\mathrm{GN}_{2 T}$, this group still held at least $90 \%$ coverage yield and a $\mathrm{I}_{\mathrm{D}} / \mathrm{I}_{G}$ ratio $<0.3$, indicating high-quality graphene (Gayathri et al. 2014). The retention of GN may be explained by the $p$ - $d$ hybridization between carbon and titanium orbitals (chemisorption) and by the diffusion of titanium atoms at the surface of graphene, creating bonds with the carbon atoms under them or occupying carbon atom vacancies (Hsu et al. 2014; Morin et al. 2017; Xu et al. 2018). Finally, similar patterns of $\mathrm{I}_{2 D} / \mathrm{I}_{G}$ and $\mathrm{FWHM}_{2 D}$ indicate that $\mathrm{GN}$ endures the microbial challenge, maintaining high structural integrity after biofilm growth and removal (Fig. 4D).

The clinical translation of GN will demand the production and transfer of graphene at large scale. Notably, CVD graphene with sizes up to $9,000 \mathrm{~cm}^{2}$ is already produced and commercially available. Although the $\mathrm{Cu}$ foil (used for the nucleation of graphene domains) accounts for $>50 \%$ of the production cost, it can be recycled to decrease the final cost (Morin et al. 2017; Zhu et al. 2018). The graphene transfer procedures are the Achilles heel, delaying several applications due to the challenges of handling a material made of 1 or few layers of atoms. Growing graphene directly on arbitrary substrates (e.g., titanium alloy) would be an ideal solution to decrease the production and transfer costs, but this cannot be achieved for all substrates since graphene films are produced under defined conditions. It is worth mentioning that the transfer step is a bottleneck deterring several industries, which have also responded by developing technologies and techniques that can increase transfer yield at lower cost (Qing et al. 2020). We hope that improvements in this critical stage will enable costeffective production and deposition of graphene on biomaterials too.

Despite the promising results, this work has limitations. The corrosion experiments under static conditions do not take into consideration additional stresses and defects that can be created by abrasion mechanical stresses and influence corrosion behavior. Further research is warranted to explore the effects of mixed microbials and the association of mechanical stresses and corrosion on GN properties.

\section{Conclusion}

The long time needed for integration to native bone and the lack of inherent antimicrobial properties warrant the development of coatings for titanium implants. GN has antiadhesive properties and enhances bone formation on titanium alloys. Here, we showed that GN maintains very high structural integrity when challenged by different physicochemical stresses relevant to the oral environment. Also, GN does not increase inflammatory responses by macrophages. Our findings open avenues for unveiling the potential of GN to accelerate osseointegration and prevent biofilm-associated diseases on implants in vivo.

\section{Author Contributions}

V. Rosa, contributed to conception, design, data analysis, and interpretation, drafted and critically revised the manuscript; R. Malhotra, S.V. Agarwalla, J.L.P. Morin, Y.M. Han, contributed to design, data acquisition, analysis, and interpretation, critically revised the manuscript; E.K. Luong-Van, contributed to conception, design, data acquisition, analysis, and interpretation, critically revised the manuscript; R.J.J. Chew, contributed to design, data acquisition, and analysis, critically revised the manuscript; C.J. Seneviratne, N. Silikas, K.S. Tan, C.A. Nijhuis, A.H. Castro Neto, contributed to design, data analysis, and interpretation, critically revised the manuscript. All authors gave final approval and agree to be accountable for all aspects of the work.

\section{Acknowledgments}

The authors acknowledge the support received from the National Research Foundation Singapore and from Yu Fan Sim for the statistical analyses.

\section{Declaration of Conflicting Interests}

The authors declared no potential conflicts of interest with respect to the research, authorship, and/or publication of this article.

\section{Funding}

The authors disclosed receipt of the following financial support for the research, authorship, and/or publication of this article: V. Rosa is supported by the grants from the Singapore Ministry of 
Education (Academic Research Fund Tier 1, R-221-000-104-114, R-221-000-132-114) and National University Health System (Open Collaborative Research Grant; October 25, 2016).

\section{ORCID iDs}

V. Rosa iD https://orcid.org/0000-0002-9203-7657

R. Malhotra iD https://orcid.org/0000-0002-6896-4063

\section{References}

Agarwalla SV, Ellepola K, Costa MCF, Fechine GJM, Morin JLP, Castro Neto AH, Seneviratne CJ, Rosa V. 2019. Hydrophobicity of graphene as a driving force for inhibiting biofilm formation of pathogenic bacteria and fungi. Dent Mater. 35(3):403-413.

Anderson JM, Rodriguez A, Chang DT. 2008. Foreign body reaction to biomaterials. Semin Immunol. 20(2):86-100.

Berry V. 2013. Impermeability of graphene and its applications. Carbon. 62:110.

Broughton G, Janis JE, Attinger CE. 2006. The basic science of wound healing. Plast Reconstr Surg. 117(7):12S-34S.

Bunch JS, Verbridge SS, Alden JS, van der Zande AM, Parpia JM, Craighead HG, McEuen PL. 2008. Impermeable atomic membranes from graphene sheets. Nano Lett. 8(8):2458-2462.

Chouirfa H, Bouloussa H, Migonney V, Falentin-Daudre C. 2019. Review of titanium surface modification techniques and coatings for antibacterial applications. Acta Biomater. 83:37-54.

Derks J, Schaller D, Hakansson J, Wennstrom JL, Tomasi C, Berglundh T. 2016. Effectiveness of implant therapy analyzed in a Swedish population: prevalence of peri-implantitis. J Dent Res. 95(1):43-49.

Duan Z. 2019. Application of graphene in metal corrosion protection. IOP Conf Ser Mater Sci Eng. 493:012020

Dubey N, Ellepola K, Decroix FED, Morin JLP, Castro Neto AH, Seneviratne CJ, Rosa V. 2018. Graphene onto medical grade titanium: an atom-thick multimodal coating that promotes osteoblast maturation and inhibits biofilm formation from distinct species. Nanotoxicology. 12(4):274-289.

Dubey N, Morin JLP, Luong-Van EK, Agarwalla SV, Silikas N, Castro Neto AH, Rosa V. 2020. Osteogenic potential of graphene coated titanium is independent of transfer technique. Materialia. 9:100604.

Fares C, Elhassani R, Partain J, Hsu S-M, Craciun V, Ren F, Esquivel-Upshaw JF. 2020. Annealing and N2 plasma treatment to minimize corrosion of SiC-coated glass-ceramics. Materials. 13(10):2375

Fretwurst T, Buzanich G, Nahles S, Woelber JP, Riesemeier H, Nelson K. 2016. Metal elements in tissue with dental peri-implantitis: a pilot study. Clin Oral Implants Res. 27(9):1178-1186.

Fukushima A, Mayanagi G, Nakajo K, Sasaki K, Takahashi N. 2014. Microbiologically induced corrosive properties of the titanium surface. J Dent Res. 93(5):525-529.

Gayathri S, Jayabal P, Kottaisamy M, Ramakrishnan V. 2014. Synthesis of few layer graphene by direct exfoliation of graphite and a Raman spectroscopic study. AIP Adv. 4:027116.

Gu M, Lv L, Du F, Niu T, Chen T, Xia D, Wang S, Zhao X, Liu J, Liu Y, et al. 2018. Effects of thermal treatment on the adhesion strength and osteoinductive activity of single-layer graphene sheets on titanium substrates. Sci Rep. $8(1): 8141$

Hsu AL, Koch RJ, Ong MT, Fang W, Hofmann M, Kim KK, Seyller T, Dresselhaus MS, Reed EJ, Kong J. 2014. Surface-induced hybridization between graphene and titanium. ACS Nano. 8(8):7704-7713.

Huang L-Y, Xu K-W, Lu J, Guelorget B, Chen H. 2001. Nano-scratch and fretting wear study of DLC coatings for biomedical application. Diamond Relat Mater. 10(8):1448-1456.

Johannsen A, Wikesjo U, Tellefsen G, Johannsen G. 2012. Patient attitudes and expectations of dental implant treatment - a questionnaire study. Swed Dent J. 36(1):7-14.
Jones JA, Dadsetan M, Collier TO, Ebert M, Stokes KS, Ward RS, Hiltner PA, Anderson JM. 2012. Macrophage behavior on surface-modified polyurethanes. J Biomater Sci Polym Ed. 15(5):567-584.

Kalbacova M, Broz A, Kong J, Kalbac M. 2010. Graphene substrates promote adherence of human osteoblasts and mesenchymal stromal cells. Carbon. 48(15):4323-4329.

Klinke T, Kneist S, de Soet JJ, Kuhlisch E, Mauersberger S, Förster A, Klimm W. 2009. Acid production by oral strains of Candida albicans and lactobacilli. Caries Res. 43(2):83-91.

Koike M, Fujii H. 2001. The corrosion resistance of pure titanium in organic acids. Biomaterials. 22(21):2931-2936.

Luong-Van EK, Madanagopal TT, Rosa V. 2020. Mechanisms of graphene influence on cell differentiation. Mater Today Chem. 16:10020.

Mabilleau G, Bourdon S, Joly-Guillou M, Filmon R, Baslé M, Chappard D. 2006. Influence of fluoride, hydrogen peroxide and lactic acid on the corrosion resistance of commercially pure titanium. Acta Biomater. 2(1):121129.

Manavathu EK, Dimmock JR, Vashishtha SC, Chandrasekar PH. 1999. Protonpumping-atpase-targeted antifungal activity of a novel conjugated styryl ketone. Antimicrob Agents Chemother. 43(12):2950-2959.

Morin JLP, Dubey N, Decroix FED, Luong-Van EK, Castro Neto AH, Rosa V. 2017. Graphene transfer to 3-dimensional surfaces: a vacuum-assisted dry transfer method. 2D Mater. 4(2):025060.

Pidhatika B, Rodenstein M, Chen Y, Rakhmatullina E, Muhlebach A, Acikgoz C, Textor M, Konradi R. 2012. Comparative stability studies of poly(2-methyl-2-oxazoline) and poly(ethylene glycol) brush coatings. Biointerphases. 7(1-4):1.

Prando D, Brenna A, Diamanti MV, Beretta S, Bolzoni F, Ormellese M, Pedeferri M. 2017. Corrosion of titanium: part 1—aggressive environments and main forms of degradation. J Appl Biomater Funct Mater. 15(4):e291e302.

Qing F, Zhang Y, Niu Y, Stehle R, Chen Y, Li X. 2020. Towards large-scale graphene transfer. Nanoscale. 12(20):10890-10911.

Qu Q, Wang L, Chen Y, Li L, He Y, Ding Z. 2014. Corrosion behavior of titanium in artificial saliva by lactic acid. Materials. 7(8):5528-5542.

Roosjen A, de Vries J, van der Mei HC, Norde W, Busscher HJ. 2005. Stability and effectiveness against bacterial adhesion of poly(ethylene oxide) coatings in biological fluids. J Biomed Mater Res B Appl Biomater. 73(2):347354.

Schönweger F, Sprecher CM, Milz S, Dommann-Scherrer C, Meier C, Dommann A, Neels A, Wahl P. 2020. New insights into osteointegration and delamination from a multidisciplinary investigation of a failed hydroxyapatite-coated hip joint replacement. Materials. 13(21):4713.

Schwarz F, Becker K, Rahn S, Hegewald A, Pfeffer K, Henrich B. 2015. Real-time PCR analysis of fungal organisms and bacterial species at periimplantitis sites. Int J Implant Dent. 1(1):9.

Souza JC, Henriques M, Oliveira R, Teughels W, Celis JP, Rocha LA. 2010a. Biofilms inducing ultra-low friction on titanium. J Dent Res. 89(12):14701475 .

Souza JC, Henriques M, Oliveira R, Teughels W, Celis JP, Rocha LA. 2010b. Do oral biofilms influence the wear and corrosion behavior of titanium? Biofouling. 26(4):471-478.

Tan KS, Nackley AG, Satterfield K, Maixner W, Diatchenko L, Flood PM. 2007. B2 adrenergic receptor activation stimulates pro-inflammatory cytokine production in macrophages via PKA- and NF- $\mathrm{kB}$-independent mechanisms. Cell Signal. 19(2):251-260.

Xie H, Cao T, Rodríguez-Lozano FJ, Luong-Van EK, Rosa V. 2017. Graphene for the development of the next-generation of biocomposites for dental and medical applications. Dent Mater. 33(7):765-774.

Xu H, Wu X, Li X, Luo C, Liang F, Orignac E, Zhang J, Chu J. 2018. Properties of graphene-metal contacts probed by Raman spectroscopy. Carbon. 127:491-497.

Zhang YY, Gu YT. 2013. Mechanical properties of graphene: effects of layer number, temperature and isotope. Comput Mater Sci. 71:197-200.

Zhu Y, Ji H, Cheng H-M, Ruoff RS. 2018. Mass production and industrial applications of graphene materials. Natl Sci Rev. 5(1):90-101. 